\title{
6-Formylindolo (3,2-b)carbazole (FICZ) enhances retinoic acid (RA)-induced differentiation of HL-60 myeloblastic leukemia cells
}

\author{
Rodica P Bunaciu and Andrew Yen ${ }^{*}$
}

\begin{abstract}
Background: The aryl hydrocarbon receptor (AhR) ligand 6-Formylindolo(3,2-b)carbazole (FICZ) has received increasing attention since its identification as an endogenous AhR ligand and a photoproduct of tryptophan. FICZ and its metabolites have been detected in human fluids. We recently reported that AhR promotes retinoic acid (RA)-induced granulocytic differentiation of HL-60 myeloblastic leukemia cells by restricting the nuclear abundance of the stem cell associated transcription factor Oct4. The standard clinical management of acute promyelocytic leukemia (APL) is differentiation induction therapy using RA. But RA is not effective for other myeloid leukemias, making the mechanism of RA-induced differentiation observed in a non-APL myeloid leukemia of interest. To our knowledge, this is the first study regarding the influence of FICZ on RA-induced differentiation in any type of leukemic blasts.

Methods: Using flow cytometry and Western blotting assays, we determined the effects of FICZ on RA-induced differentiation of HL-60 human leukemia cells. All experiments were performed in triplicate. The groups RA and FICZ + RA were compared using the Paired-Samples T-Test. Western blot figures present the typical blots.

Results: We demonstrate that FICZ enhances RA-induced differentiation, assessed by the expression of the membrane differentiation marker CD11b; cell cycle arrest; and the functional differentiation marker, inducible-oxidative metabolism. FICZ causes changes in signaling events that are known to drive differentiation, and notably augments the RA-induced sustained activation of the RAF/MEK/ERK axis of the mitogen-activated protein kinase (MAPK) cascade. FICZ also augments expression of the known MAPK signaling regulatory molecules c-Cbl, VAV1, pY458 p85 PI3K, Src-family kinases (SFKs), and IRF-1, a transcription factor associated with this putative signalsome that promotes RA-induced differentiation. Moreover, FICZ in combination with RA also increases expression of AhR and even more so of both Cyp1A2 and p47phox, which are known to be transcriptionally regulated by AhR. pY1021 PDGFRß, a marker associated with retinoic acid syndrome was also increased.
\end{abstract}

Conclusions: Our data suggest that FICZ modulates intracellular signaling pathways and enhances RA-induced differentiation.

Keywords: Retinoic acid (RA), 6-Formylindolo(3,2-b)carbazole (FICZ), Differentiation, Neutrophil, HL-60

\footnotetext{
* Correspondence: ay13@cornell.edu

Department of Biomedical Sciences, Cornell University, Ithaca, NY 14853, USA
}

\section{Ciomed Central}

(c) 2013 Bunaciu and Yen; licensee BioMed Central Ltd. This is an Open Access article distributed under the terms of the Creative Commons Attribution License (http://creativecommons.org/licenses/by/2.0), which permits unrestricted use, distribution, and reproduction in any medium, provided the original work is properly cited. 


\section{Background}

Retinoic acid (RA) induces leukemic cell differentiation in a process that depends on AhR [1]. AhR overexpression drives differentiation [1]. This motivates interest in the effects of an endogenous AhR ligand on this process. AhR is a ligand activated receptor. There are two intensely studied AhR functions, both being ligand dependent. AhR is a basic helix-loop-helix/Per-Arnt-Sim (bHLH-PAS) transcription factor [2], and also an adaptor in the cullin 4B ubiquitin ligase complex [3]. It has been found to be expressed in all tissues analyzed. It is present in the cytosol and in the nucleus. Its transcriptional activity is the most studied, especially its regulation of detoxification enzymes such as cytochrome P450 [4]. The RAR/RXR and AhR pathways are known to crosstalk. For example, they compete for the silencing mediator of retinoid and thyroid receptors (SMRT) protein [5]. Consistent with various molecular indications of crosstalk, the two pathways can give rise to similar pathologies. For example, teratogenic effects such as cleft palate and hydronephrosis can be induced by retinoids [6] and also by an AhR agonist, 2,3,7, 8-tetrachlorodibenzo-p-dioxin (TCDD) [7]. They can also contribute to common developmental processes. For example, in fish, RA and its receptors are required both for AhR transcription and embryonic development of blood vessels and bones [8]. AhR can thus regulate RA effects, as well as vice versa, but the mechanisms are not well understood.

Recently, several papers reported that the AhR gene can act as a tumor suppressor in the absence of xenobiotics. AhR has been shown to have a role in propelling breast cancer [9] and liver cancer [10] cell differentiation. AhR knockout mice injected with the liver tumor initiator diethylnitrosamine (DEN) have increased liver tumor formation and growth, with increased cell proliferation, inflammatory cytokine expression and DNA damage compared to wild type mice treated with DEN or untreated mice [10]. Moreover, the AhR knockout mice have increased cecal carcinogenesis [11]. Certain AhR antagonists promote hematopoietic stem cell proliferation [12]. The full molecular mechanism of AhRdependent tumor suppressing activity is far from being elucidated; however, some details are emerging. Historically, the most studied function of AhR is its transcriptional activity elicited by xenobiotics. Recently it has become apparent that xenobiotics and endogenous ligands have different transcriptional properties, leading to opposite outcomes. For example, it was proposed that transient AhR transcriptional activity, characteristic of endogenous ligands such as 6-Formylindolo(3, 2-b)carbazole (FICZ), is essential for the role of AhR in stem/progenitor cell homeostasis, whereas prolonged transcriptional activation is induced by exogenous ligands, such as TCDD, a well known carcinogen [13].
The more recently emerging role of AhR in protein degradation via CUL4B/AhR-mediated ubiquitylation and consequently cancer suppression is also of potentially related significance [11]. While the mechanisms are not yet clear, it appears that depending on the model system and on the ligand used, AhR can drive transformation or differentiation.

We have previously shown that AhR propels RAinduced differentiation of human myeloblastic leukemia cells by downregulating the nuclear transcription factor, Oct4 [1]. Oct4 is a Yamanaka-Thomson factor controlling stem cells [14-16]. This process depends on MAPK signaling. This motivates interest in the effect of endogenous AhR ligands, such as FICZ, on the MAPK pathway and its associated signaling events known to drive RA-induced differentiation. Unlike transcription, the effects of FICZ on signaling are less explored and remain to be better described.

One well studied model of leukemic cell differentiation is HL-60. HL-60 is a human myeloblastic leukemia cell line that is lineage uncommitted and capable of granulocytic or monocytic differentiation in response to different agents. HL-60 is a NCI-60 line, a set of standard cell lines, used for example in drug testing. It has been extensively used as a model for pharmacologically induced differentiation. HL60 cells undergo granulocytic differentiation with G0/G1 growth arrest when treated with RA. This process requires sustained activation of MAPK signaling along the RAF/ MEK/ERK axis [17], and a cascade of signaling regulatory events involving Src-family kinases, c-Cbl, VAV1, PI3K, and IRF-1 [18-22]. During RA-induced differentiation, ectopic expression of interferon regulatory factor 1 (IRF-1) [21] and c-Cbl [19] have been shown to enhance ERK 1/2 activation and promote RA-induced differentiation and G0/G1-arrest. The VAV1 guanine nucleotide exchange factor implicated in myelopoiesis also was reported to promote RA-induced granulocytic differentiation [23].

The present study demonstrates that FICZ is able to augment RA-induced differentiation. FICZ increases the amount and activation of key components of the MAPK signaling cascade known to drive differentiation, and this signaling modulation is consistent with a ligand bound AhR dependence as demonstrated by using the classical pharmacological AhR agonist $\beta$-naphthoflavone ( $\beta$-NF) and antagonist $\alpha$-naphthoflavone $(\alpha-\mathrm{NF})$. These had positive and negative effects on the signaling events consistent with their AhR agonist vs. antagonist activity. The findings suggest a novel potential mechanism of collaboration between RA and FICZ during RA-induced differentiation of $t(15 ; 17)$ negative leukemic blasts.

\section{Results and discussion}

The capability to prevent and treat leukemia depends upon understanding the molecular underlying mechanisms of 
(a) pathogenesis, (b) induction of differentiation and apoptosis and (c) resistance to therapy. Multiple pathways are involved in each of these three aspects; however the aryl hydrocarbon receptor (AhR) is strikingly involved in all three of the above mentioned phenomena. We have shown that during RA-induced differentiation, AhR propels differentiation [1]. We now sought evidence on whether FICZ, an endogenous AhR ligand in humans, affects RAinduced leukemic cell differentiation.

\section{FICZ augments RA-induced differentiation markers}

To determine if FICZ influenced RA-induced differentiation, HL-60 cells were treated with both agents either alone or in combination, and consequential occurrence of differentiation markers was measured. RA-induced granulocytic differentiation is characterized by the appearance of several phenotypic differentiation markers. These include: cell surface CD11b, cell cycle arrest in G0/G1, and inducible respiratory burst - a classical functional differentiation marker that is a characteristic response of mature myeloid cells to bacterial cell components. FICZ (100 nM) by itself had no effect on these markers. Co-administered with RA, FICZ enhanced the induced expression of these markers compared to RA alone.

Cells were untreated or treated with $1 \mu \mathrm{M}$ RA with or without 100 nM FICZ. Expression of the CD38 and CD11b

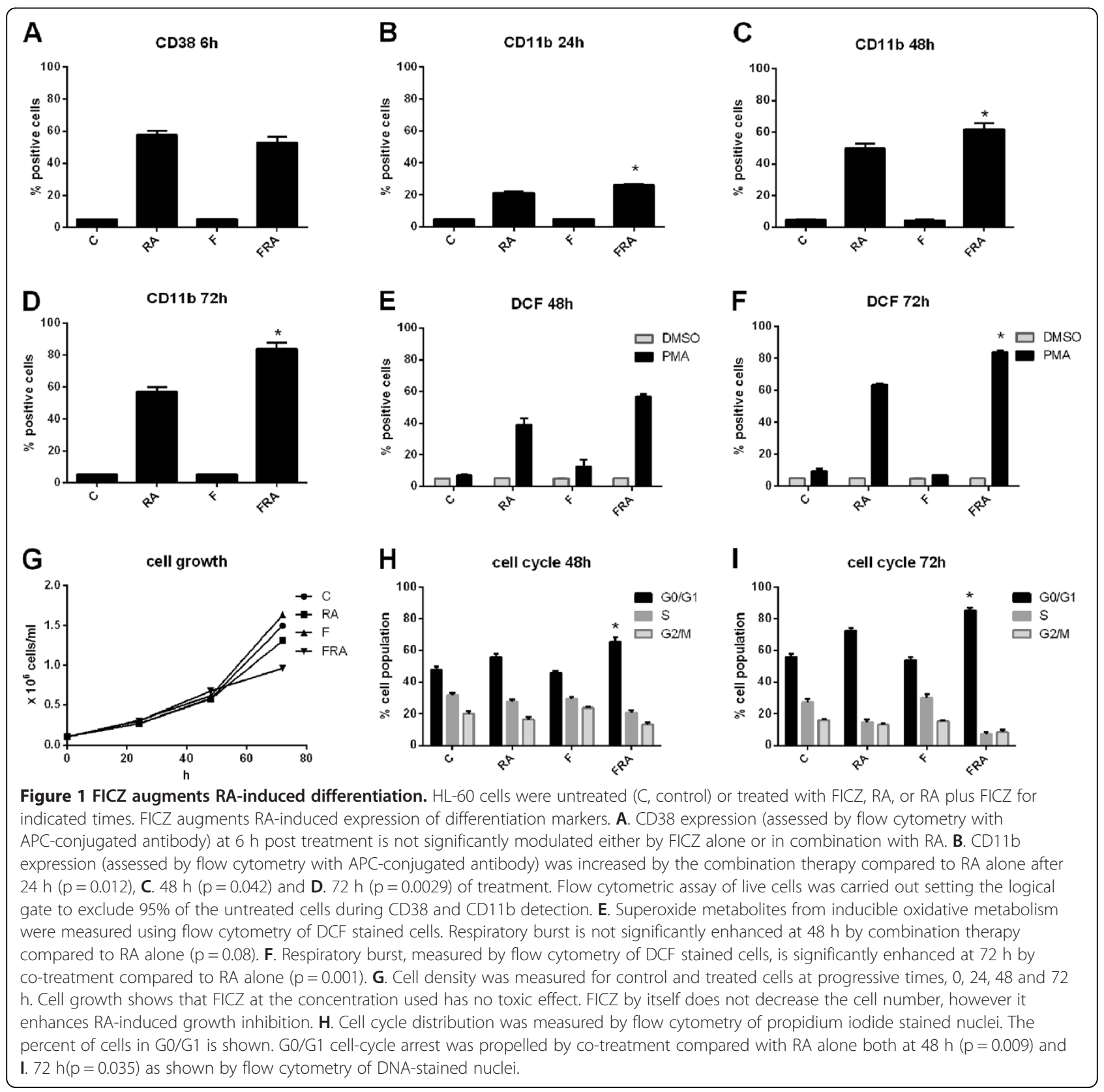


cell surface differentiation markers, the respiratory burst (measured as inducible reactive oxygen species) and the percentage of cells with G0/G1 DNA were measured by flow cytometry (Figure 1). CD38 is an early cell surface differentiation marker. At $6 \mathrm{~h}$, FICZ alone did not induce CD38 expression. Likewise, FICZ did not affect RA-induced CD38 expression at this early time (Figure 1A). $\mathrm{CD} 11 \mathrm{~b}$ is the alpha subunit of the integrin receptor and is a differentiation marker that typically appears with slower kinetics than CD38 in RA-treated cells. For CD11b expression, the percentage of cells that were positive was higher for cells treated with RA plus FICZ compared to RA alone, namely $26 \%$ versus $21 \%, \mathrm{p}=0.012$ after $24 \mathrm{~h}$ (Figure $1 \mathrm{~B}$ ), $62 \%$ versus $50 \%, \mathrm{p}=0.042$, after $48 \mathrm{~h}$ (Figure $1 \mathrm{C}$ ) and $84 \%$ versus $57 \%, \mathrm{p}=0.0029$, after $72 \mathrm{~h}$ (Figure 1D). The flow cytometry raw data and mean fluorescence index for a representative experiment ( $48 \mathrm{~h}$ and $72 \mathrm{~h}$ ) are presented in Additional file 1: Figure S1. Cells treated with FICZ alone showed no CD11b expression - like untreated controls.

Inducible oxidative metabolism is a functional marker of further differentiation that is characteristic of mature cells. This mature functional differentiation marker was also enhanced in cells treated with FICZ plus RA compared to RA alone. At 48 h, FICZ plus RA treated cells were $57 \%$ positive compared to $39 \%$ for cells treated with RA alone with a $\mathrm{p}=0.08$ (Figure $1 \mathrm{E}$ ), and by $72 \mathrm{~h}$ $84 \%$ of FICZ plus RA treated cells were positive versus $63 \%$ of RA treated cells with a $\mathrm{p}=0.001$ (Figure 1F).

G0/G1 cell cycle arrest is a characteristic of differentiation. RA caused an increase in the relative number of G0/G1 cells and an associated reduction in $S$ phase cells. Addition of FICZ with RA enhanced this effect (Figure 1H and $1 \mathrm{I})$, consistent with the enhanced phenotypic shift. At 48h (Figure 1H), 48\% cells were in G0/G1 phase for untreated cells, and $56 \%$ for RA treated cells, $\mathrm{p}<0.0001$. At $72 \mathrm{~h}$ (Figure 1I), the proportions were $56 \%$ and $72 \%$ for untreated and RA treated respectively $(\mathrm{p}<0.001)$. FICZ alone had a slightly lower proportion of cells in G0/G1 compared to untreated cells ( $46 \%$ vs. $48 \%, \mathrm{p}=0.2$ at $48 \mathrm{~h}$, and $54 \%$ vs. $56 \%, \mathrm{p}=0.01$ at $72 \mathrm{~h}$ ). For cells treated with FICZ plus RA compared to RA alone, the percentage of cells with G0/G1 DNA was 66\% compared to 56\%, p < 0.0001 , after $48 \mathrm{~h}$; and $85 \%$ versus $72 \%, \mathrm{p}<0.0001$, after 72 h. Growth curves (Figure 1G) were consistent with the cell cycle phase distribution changes. FICZ alone did not significantly affect, although slightly increased, the cell density compared with control $\left(1.5 \pm 0.08 \times 10^{6} \mathrm{cells} / \mathrm{ml}\right.$ for control, and $1.6 \pm 0.1 \times 10^{6}$ cells $/ \mathrm{ml}$ for FICZ at $72 \mathrm{~h}$ post-treatment). FICZ in combination with RA lowered the cell densities compared to RA alone $\left(0.96 \pm 0.15 \times 10^{6}\right.$ cells $/ \mathrm{ml}$ for the combination treatment and $1.3 \pm 0.1 \times 10^{6}$ cells/ml for RA alone at $72 \mathrm{~h}$ post-treatment) consistent with the G0/G1 data. FICZ thus enhances RA-induced CD11b expression, inducible oxidative metabolism, and
G0/G1 arrest, but does not modulate these parameters by itself in the absence of RA. FICZ caused no evident toxicity, evaluated by trypan blue exclusion or population growth, and FICZ-treated cells had similar cell cycle phase distribution and growth curves as untreated control cells.

Given the positive effects of FICZ on RA-induced differentiation, we sought evidence that the FICZ as presented in this context could regulate the transcriptional activity of AhR by determining its effects on two classical AhR transcriptionally regulated targets: Cyp1A2 and p47phox.

\section{FICZ augments the expression of classical AhR transcriptionally regulated genes}

The expression of cytochrome P450 1A2 (Cyp1A2), neutrophil cytosolic factor-1 (p47phox), and aryl hydrocarbon receptor (AhR), were analysed after $48 \mathrm{~h}$ of treatment with FICZ, RA or their combination using Western blotting (Figure 2). We found that relative levels of Cyp1A2 and p47phox proteins were clearly increased by the combination therapy (lane 4) compared with untreated control cells (lane 1). Addition of FICZ to RA (lane 4) also increased Cyp1A2 and p47phox expression compared to RA only-treated cells (lane 2). Cyp1A2, an endogenous reporter of classical AhR driven transcriptional activation thus behaved as expected. RA alone did not induce Cyp1A2 expression, and FICZ induced it both alone and more strongly with RA. The protein $\mathrm{p} 47$ phox, a NADPH oxidase subunit of the complex producing the respiratory burst, was also reported to be under AhR transcriptional control [24]. In contrast to Cyp1A2, the changes in p47phox expression depended on the presence of RA. FICZ was able to upregulate p47phox expression only in RA-treated cells. This was anticipated since p47phox expression is a characteristic of mature myeloid cells, and RA is needed to cause granulocytic differentiation. AhR expression was modestly increased by RA plus FICZ (lane 4) compared to RA alone (lane2). Previous reports showed that AhR protein expression is augmented by treatment with RA [1] or FICZ alone [25] and we confirmed this (lane 2 vs lane 1 or lane 3 vs lane 1). FICZ thus increases the expression of genes that are classical targets of AhR. While the present results are consistent with action through AhR, there could be a variety of other transcription factors that also contribute to the FICZ induced effects observed.

It is now well established that a transient activation of the MAPK signaling cascade elicits cell proliferation, whereas prolonged activation leads to differentiation $[17,26]$. In particular RAF activation is known to drive RA-induced differentiation [27]. We therefore assessed the effects of FICZ on the MAPK cascade, specifically the RAF/MEK/ERK axis that is activated during RAinduced differentiation. 


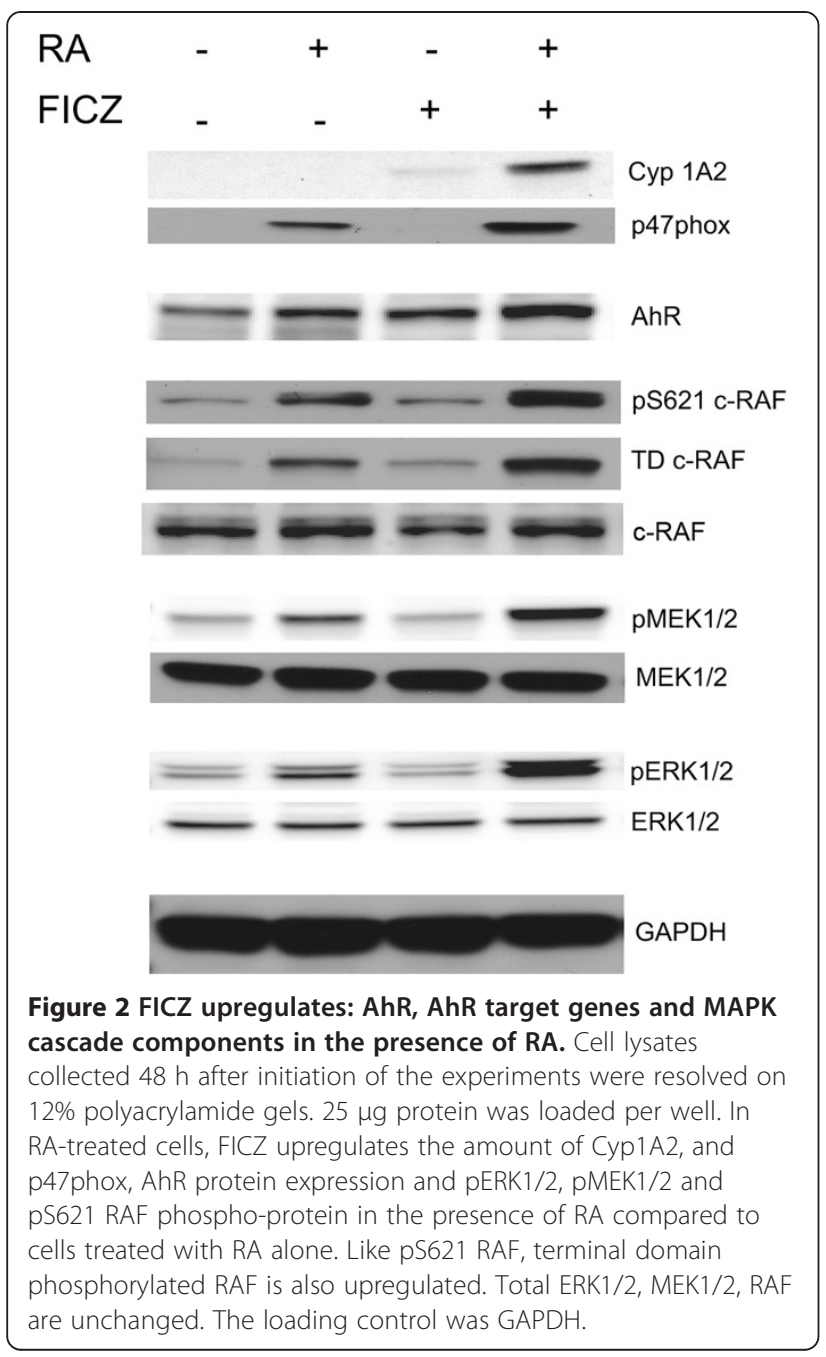

FICZ augments RA-induced MAPK signaling cascade MAPK signaling during RA-induced differentiation utilizes c-RAF activation, specifically pS621 c-RAF phosphorylation, which is necessary to induce terminal granulocytic differentiation [27]. Western blot analysis confirms that FICZ and RA co-treatment enhances c-RAF activation (Figure 2) compared to RA alone. FICZ alone had no effect. The same behavior is true for the other two components of the MAPK cascade: pMEK1/2 and pERK1/2. Total amounts of c-RAF, MEK, and ERK in contrast were not upregulated in this time frame $(48 \mathrm{~h})$ by FICZ or FICZ plus RA. The data thus indicate FICZ regulates intracellular signaling events, but not c-RAF, MEK or ERK abundance - such as might occur through AhR regulated transcription or protein stability. Interestingly, FICZ and RA co-treatment also resulted in increased phospho-cRAF pS289/296/301 (TD RAF) compared to RA alone. This C-terminal domain of c-RAF is phosphorylated during RA-induced differentiation and is thought to be part of a putative feedback loop characterizing hyperactive
MAPK signaling needed for differentiation [18]. In other contexts, it is also known to be phosphorylated by ERK $1 / 2$ [28] and can make the c-RAF molecule unresponsive to further stimulation [29], suggesting that this phosphorylation event may have a diversity of potential effects dependent on context. FICZ thus augments the RA-induced activation of the RAF/MEK/ERK axis. The enhanced activation is consistent with the occurrence of enhanced differentiation attributed to FICZ above.

The MAPK signalsome that drives RA-induced differentiation is known to contain a number of regulatory molecules that propel differentiation. We thus sought evidence of their involvement consequential to FICZ. Interestingly, the signalsome has been found to contain the transcription factor IRF-1 which has also been found to propel RA-induced differentiation [21].

MAPK signaling cascade modulation by FICZ is consistent with modulation of other signalsome regulatory molecules of the RA-induced differentiation process c-Cbl and IRF-1 have been previously shown to be instrumental in RA-induced differentiation; specifically, increased expression propelled differentiation [21]. Cells were treated with RA or FICZ alone or in combination, and expression of c-Cbl, pY507 Lyn, RAR $\alpha$, IRF-1 and pY1021 PDGFR $\beta$ was measured. FICZ augments the RA-induced increases in c-Cbl and IRF-1 (Figure 3). This is consistent with previous results where we have shown that AhR expression induced IRF-1, and IRF-1 physically interacted with c-Cbl [21]. To confirm that the increases in amount of protein that we observe are not attributable to a general nonspecific increase in protein synthesis, we have confirmed that the amount of RAR $\alpha$ or GAPDH did not increase. Lyn is a member of the Src-family kinases (SFKs), and its binding to c-RAF in RA treated cells is enhanced by the SFK inhibitor PP2, which enhanced RA-induced differentiation [18]. We reported that a scaffolding function of Lyn - not its kinase activity - was important for RA-induced differentiation [18]. Phosphorylation of Lyn at Y507 increases autoinhibition of its kinase activity [30]. RA increases the amount of pY507 Lyn and addition of FICZ augments this, again consistent with a role of FICZ in enhancing RA-induced effects on signaling molecules. We also assessed pY1021 PDGFR $\beta$ expression. pY1021 PDGFR $\beta$ is potentially significant as a marker of neutrophil hyperactivation, consistent with the report that pY1021 PDGFR $\beta$ is a marker of retinoic acid syndrome [31]. It was also up regulated by RA, and addition of FICZ to the RA further enhanced it. FICZ thus enhanced RA effects on a number of RA-targeted signaling regulatory molecules associated with induced differentiation.

We sought evidence to corroborate the putative action of FICZ through AhR to drive signaling effects by using other known AhR agonists and antagonists. 


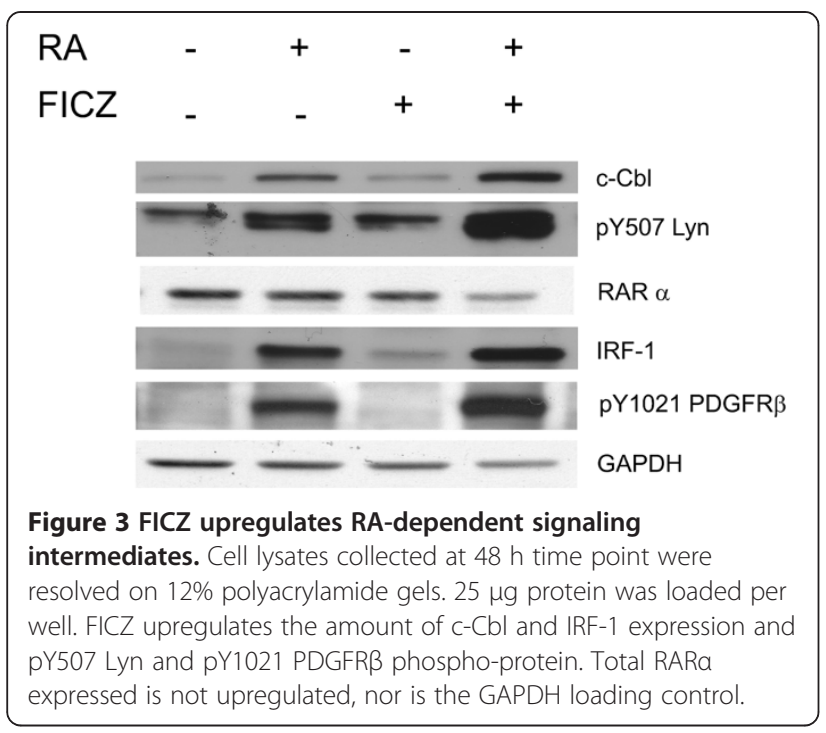

The effects of other AhR ligands on signaling

The ability of FICZ to modulate signaling molecules in the context of RA-treated cells is novel. FICZ is an endogenous AhR ligand. This motivated interest in determining if other AhR ligands also had consistent effects on signaling. Two well characterized exogenous AhR ligands were used: an AhR antagonist, $\alpha-\mathrm{NF}$, and an agonist, $\beta-\mathrm{NF}$, at a concentration of $1 \mu \mathrm{M}$ each. Cells were treated with RA, FICZ, $\alpha-N F$ or $\beta-N F$ as shown in the figures. The effects on Cyp1A2, TD RAF and pS621 c-RAF were measured by Western blotting as shown in Figure 4. Cyp1A2 is a classical responder to AhR activation and was used to confirm the ability of the ligands to activate AhR or not. FICZ increases Cyp1A2 expression and behaves as an AhR agonist as expected. At the concentration used $\beta-\mathrm{NF}$ elicits Cyp1A2 expression also, whereas $\alpha$-NF does not, consistent with their known roles as an AhR agonist or antagonist, respectively. RA augments the effects of the AhR agonists (FICZ or $\beta-N F$ ), but not the antagonist. This suggests cooperativity between RA and the agonists (with lanes 4 and 8 of Figure 4 having the strongest signals). We next determined if there were corresponding cooperative effects on signaling events believed to drive RAinduced differentiation. RA-induced upregulation of the C-terminal domain phosphorylated RAF (TD-RAF), and this is enhanced by the AhR agonists (FICZ and $\beta-N F$ ), but not by the antagonist $(\alpha-N F)$. There are similar but more subtle effects on the expression of pS621 c-RAF. RA and the agonists again cooperate, and pS621 c-RAF expression is greater for RA plus agonist than RA alone. Both the C-terminal domain and S621 c-RAF phosphorylations are characteristic of RA-induced signaling. Hence the TD RAF and pS621 c-RAF responses to RA are augmented by AhR agonists.

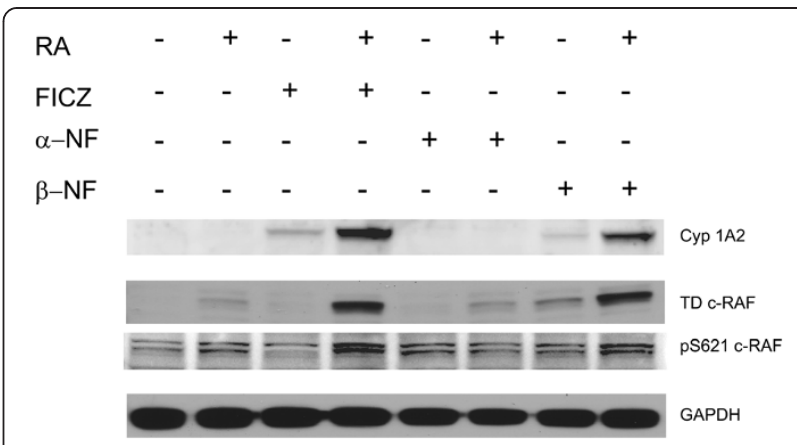

Figure 4 AhR ligands modulate phosphorylation of RAF in the MAPK signaling cascade. Cell lysates collected $48 \mathrm{~h}$ after initiation of the experiments were resolved on $12 \%$ polyacrylamide gels. $25 \mu \mathrm{g}$ protein was loaded per well. $\beta$-NF upregulates the amount of Cyp1A2, TD RAF and pS621RAF proteins in the presence of RA in a similar fashion to FICZ (comparing similarity of lanes 4 and 8), whereas a-NF does not. GAPDH is the loading control.

The RA-regulated RAF/MEK/ERK axis has been found to be associated with a number of signaling regulatory molecules in a putative signalsome that propels RA-induced differentiation. Prominent MAPK signaling regulators in the RA-induced signaling cascade leading to RA-induced differentiation that have emerged are: Src-family kinases [18], VAV1 [23,32] and PI3K [22]. Cells were treated with RA or the antagonist or agonists singly or in combination with RA as above and the expression of these targeted signaling molecules was measured. The protein levels and activation of these signaling molecules are modulated during RA-induced differentiation by AhR ligands (Figure 5). Fgr, a SFK, is one of the most responsive of these proteins. RA-induced upregulation of Fgr is enhanced by FICZ and $\beta-N F, A h R$ agonists, but is crippled by $\alpha-N F$, an AhR antagonist. The AhR ligands by themselves had no discernible effect on expression, indicating an AhR role dependent on the RA-induced context. Consistent with this, the enzymatically active form of SFKs discerned by probing with a p-Y416 pan-Src-family kinase antibody also responded similarly to the AhR ligands as seen for Fgr expression. The results are consistent with earlier observations for these cells that the SFKs are progressively activated by tyrosine phosphorylation after RA treatment and reach maximum phosphorylation 48 hours post treatment [33]. Taken together, these observations are thus consistent with a role for these kinases in driving differentiation. Interestingly, the pY507 Lyn is also regulated likewise by RA and the AhR agonists and antagonist (lanes 4 and 8 Figure 5 show the strongest signal again). This phosphorylation site has been implicated with a negative role in another context [34]. This is consistent with a previous suggestion that Lyn performs a scaffold function important for the signalsome to drive differentiation [18]. The total amount of Lyn, the VAV1 guanine nucleotide exchange factor and pY458 p85 PI3K also exhibit a similar enhancement after RA plus 


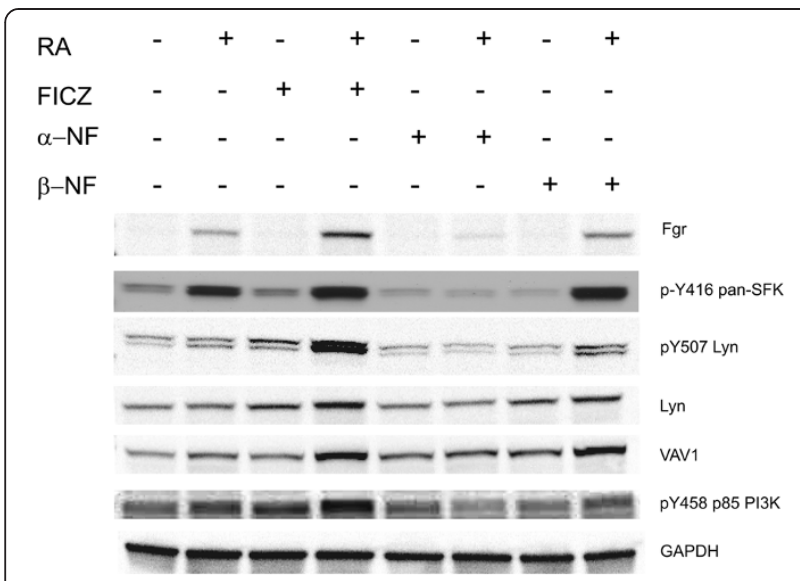

Figure 5 FICZ and other AhR ligands modulate MAPK signaling cascade regulatory molecules. Cell lysates collected after $48 \mathrm{~h}$ of RA treatment were resolved on $12 \%$ polyacrylamide gels. $25 \mu \mathrm{g}$ protein was loaded per well. Similarly to FICZ, $\beta$-NF upregulates the amount of Fgr, Lyn, VAV1 protein and p-Y416 pan-SFK, pY507 Lyn, and pY458 p85PI3K phospho-protein, whereas the antagonist does not upregulate those markers Comparing lanes 4 and 8 FICZ and $\beta-N F$, the two AhR agonists, have apparently similar signaling profiles in RA-treated cells, which is not shared by a-NF, an AhR antagonist, lane 6). The agonists by themselves without RA do not have this effect. GAPDH is the loading control.

FICZ combination treatment compared to RA alone. However the effects of $\alpha-\mathrm{NF}$ and $\beta$-NF on total Lyn, VAV1, and pY458 p85 PI3K are minor compared to the modulation of Fgr, p-Y416 pan-SFK and pY507 Lyn. In sum, our results show that the effects of the $\beta$-NF agonist and $\alpha-\mathrm{NF}$ antagonist on certain signaling molecules that are implicated as part of the RA-regulated MAPK signaling complex are consistent with a role for AhR in driving FICZ augmentation of RA-induced differentiation via effects on components of the putative signalsome. However, some components are more responsive than others.

\section{Conclusions}

Taken together our results show that FICZ enhances RAinduced differentiation as evidenced by CD11b membrane receptor expression, inducible respiratory burst, G0/G1 cell cycle arrest and growth curves. Upregulation of signaling molecules previously shown to drive RA-induced differentiation was enhanced by addition of FICZ. Specifically, FICZ augments RAF/MEK/ERK axis MAPK signaling, Src-family kinase activation and the RA-dependent upregulation of VAV1, c-Cbl, pY458 p85PI3K, and IRF-1 expression. Interestingly, in the case of PDGFR which differentially regulates different aspects of RA-induced differentiation [35], PDGFR $\beta$ phosphorylation at Tyr 1021, a response associated with mimicking retinoic acid syndrome in this model [31], was also enhanced by the RA plus FICZ combination treatment compared to RA treatment alone.
AhR is involved in both promoting and inhibiting proliferation. AhR has been implicated with historically wellknown pro-proliferative functions. For example, benzene, an AhR agonist, is known to induce both leukemia and multiple myeloma [36]. But AhR has also been shown to have a role in propelling breast cancer [9] and liver cancer [10] cell differentiation. The present results suggest that RA may set a context for AhR to act in an antiproliferation pro-differentiation capacity. Here we provide evidence for a novel way of using an endogenous AhR ligand to enhance RA-induced differentiation associated with the unanticipated modulation of components of the MAPK and Src-family kinase signaling machine/signalsome thought to drive RA-induced differentiation (summarized in Figure 6).

The present results suggest cooperative crosstalk between the RA and FICZ elicited pathways in driving differentiation. How this occurs molecularly is a matter of conjecture that will require further experimental elucidation. There are numerous pathways that RA and FICZ are able to elicit. The most studied are RAR/RXR and AhR transcriptional regulation pathways. There are several ways those pathways are known to crosstalk. (1) For example, they compete for transcriptional co-activators/repressors, such as SMRT protein [5,37]. However, in our case, the amount of SMRT that co-immunoprecipitates with AhR does not vary with different treatments (data not shown), suggesting that this is not the mechanism involved in this case. (2) RAR and AhR can also use the same coactivators, specifically SRC-1, steroid coactivator-1 [38,39]. (3) Retinoids are reported to be AhR ligands that can drive AhR/ARNT to xenobiotic response elements (XRE) and consequently regulate transcription [5]. (4) RAR and AhR pathways can also crosstalk by regulating the same transcription factor, notably the pro-proliferation transcription factor AP-1. RAR can physically bind either c-jun or c-fos resulting in a mutual inhibition of DNAbinding activity for both RAR and AP-1 [40]. AhR is also reported to inhibit AP-1 DNA binding activity [41]. (5) RAR and AhR regulation of transcription can depend on common transcription factors such as the COUP orphan receptors which are regulators of both AhR [42] and of RAR directed transcriptional activity [43]. There are thus a variety of ways that RA and AhR governed pathways can converge at the level of transcription.

While crosstalk at the level of transcriptional regulation is arguably the most prominently studied, nonnuclear/cytoplasmic interactions at the level of signaling are also indicated. RA itself can regulate MAPK related signaling molecules such as PKC $[44,45]$ or c-RAF [46] as a lipid interacting molecule with a hydrophobic pocket $[47,48]$. AhR can also regulate pathways incorporating MAPK signaling molecules $[1,49]$. AhR has been found complexed with Src, a well-known MAPK signaling 


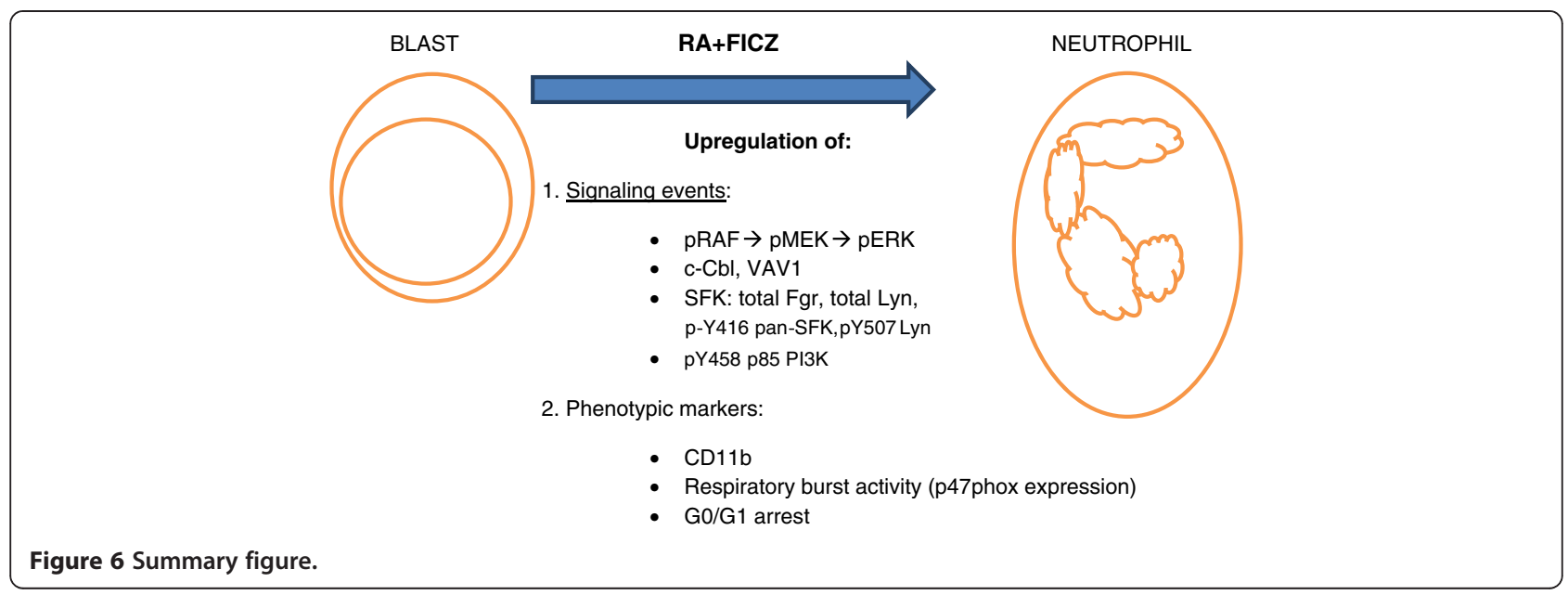

regulator [50]. And MAPK signaling has been shown to be a downstream effector for both RA and AhR, consistent with the possibility that RA and AhR integrate their cytoplasmic signaling through the MAPK axis $[1,17]$. AhR is also known to have a ubiquitin E3 ligase activity that can affect expression levels of other molecules, notably ER which we have reported can act as a membrane receptor in addition to its historical nuclear function as a ligand activated transcription factor - that originates MAPK signaling relevant to RA-induced differentiation [51]. There are thus a number of possibilities for the mechanism of non-nuclear as well as nuclear crosstalk already suggested in the literature. The present results motivate interest in deciphering their roles in RA-induced differentiation augmented by FICZ.

RA has clinically been notably successful in inducing remissions, albeit transient, in APL, but has not been effective in other myeloid leukemias. APL is defined by the presence of the PML-RAR $\alpha$ fusion protein resulting from the $t(15 ; 17)$ translocation that cytogenetically characterizes the disease, which is a FAB M3. There is thus potential interest from the therapeutic point of view of bringing RA differentiation induction therapy to nonAPL FAB M2 or 1 disease. In particular mechanistic aspects of how a FAB M2 derived cell that is capable of RA-induced differentiation undergoes granulocytic differentiation and G0 cell cycle arrest may provide insights into how to drive differentiation in a non-APL cell. Such is HL-60, the currently used model derived from a myeloblastic leukemia. Hence means of driving RA-induced differentiation here may contribute insights of therapeutic relevance.

\section{Methods}

\section{Cell culture and treatments}

HL-60 human myeloblastic leukemia cells derived from the original patient isolate, a generous gift of Dr. Robert Gallagher, were grown in RPMI 1640 (Invitrogen, Carlsbad,
CA) supplemented with $5 \%$ fetal bovine serum (Hyclone, Logan, UT) and $1 \mathrm{x}$ antibiotic/antimycotic (Sigma, St. Louis, $\mathrm{MO}$ ) in a $5 \% \mathrm{CO}_{2}$ humidified atmosphere at $37^{\circ} \mathrm{C}$. The cells were cultured in constant exponential growth as previously described [52]. The experimental cultures were initiated at a density of $0.1 \times 10^{6}$ cells $/ \mathrm{ml}$. Viability was monitored by $0.2 \%$ trypan blue (Invitrogen, Carlsbad, CA) exclusion and routinely exceeded 95\%. All reagents were purchased from Sigma (St Louis, MO) unless otherwise stated.

For treatments, all-trans-retinoic acid (RA) (Sigma, St. Louis, MO) was added from a $5 \mathrm{mM}$ stock solution in $100 \%$ ethanol to make a final concentration of $1 \mu \mathrm{M}$ in culture. 6-Formylindolo(3,2-b)carbazole (FICZ) (Enzo Life Sciences, Exeter, United Kingdom), was added from a 100 $\mu \mathrm{M}$ DMSO stock to make a final concentration of $100 \mathrm{nM}$ in culture. The concentration was chosen from an initial dose response experiment as the lower concentration yielding a phenotypic response when added with RA with no toxic effects. This corresponds to a frequently used concentration in the literature. $\alpha$-naphthoflavone and $\beta$ naphthoflavone (both from Sigma, St. Louis, MO) were each used at a final concentration of $1 \mu \mathrm{M}$ in culture. The stock solutions were $1 \mathrm{mM}$ in DMSO. Similar to FICZ, there was no apparent toxicity of $\alpha-\mathrm{NF}$ or $\beta-\mathrm{NF}$ at this dose discernible by proliferation rates, cell cycle distribution, or trypan blue exclusion.

\section{CD38, CD11b quantification}

Expression of cell surface differentiation markers was quantified by flow cytometry. $1 \times 10^{6}$ cells were collected from cultures and centrifuged at $1000 \mathrm{rpm}$ for 5 min. Cell pellets were resuspended in $200 \mu \mathrm{l}, 37^{\circ} \mathrm{C}$, PBS containing $2.5 \mu \mathrm{l}$ of allophycocyanin (APC) conjugated antibody for CD11b or CD38 (both from BD Biosciences, San Jose, CA). Following a $1 \mathrm{~h}$ incubation at $37^{\circ} \mathrm{C}$ cell surface expression levels were analyzed with a BD LSRII flow cytometer (BD Biosciences, San Jose, CA). 
APC is excited at $633 \mathrm{~nm}$ and emission collected with a $660 / 20$ band pass filter. Undifferentiated control cells were used to determine the fluorescence intensity of cells negative for the respective surface antigen. The gate to determine percent increase of expression was set to exclude $95 \%$ of the control population.

\section{Respiratory burst quantification}

Respiratory burst (a functional differentiation marker for mature myelo-monocytic cells) was measured by flow cytometry. $1 \times 10^{6}$ cells were collected and centrifuged at $1000 \mathrm{rpm}$ for $5 \mathrm{~min}$. Cell pellets were resuspended in 500 $\mu \mathrm{l} 37^{\circ} \mathrm{C}$ PBS containing $5 \mu \mathrm{M}$ 5-(and-6)-chloromethyl$2^{\prime}, 7^{\prime}$-dichlorodihydro-fluorescein diacetate acetyl ester (H2-DCF, Molecular Probes, Eugene, OR) and $0.2 \mu \mathrm{g} / \mathrm{ml}$ 12-o-tetradecanoylphorbol-13-acetate (TPA, Sigma, St. Louis, MO). H2-DCF and TPA stock solutions were made in DMSO (Sigma, St. Louis, MO) at concentrations of 0.2 $\mathrm{mg} / \mathrm{ml}$ and $5 \mathrm{mM}$, respectively. A control group incubated in H2-DCF and DMSO only was included. Cells were incubated for $20 \mathrm{~min}$ at $37^{\circ} \mathrm{C}$ prior to analysis by flow cytometry. Oxidized DCF was excited by a $488 \mathrm{~nm}$ laser and emission collected with a 530/30 nm band pass filter. The shift in fluorescence intensity in response to TPA was used to determine the percent cells with the capability to generate inducible oxidative metabolites [36]. Gates to determine percent positive cells were set to exclude $95 \%$ of control cells not stimulated with TPA.

\section{Cell cycle quantification}

$1 \times 10^{6}$ cells were collected by centrifugation and resuspended in $200 \mu \mathrm{l}$ of cold propidium iodide (PI) hypotonic staining solution containing $50 \mu \mathrm{g} / \mathrm{ml}$ propidium iodine, $1 \mu \mathrm{l} / \mathrm{ml}$ Triton X-100, and $1 \mathrm{mg} / \mathrm{ml}$ sodium citrate (all from Sigma, St. Louis, MO). Cells were incubated at room temperature for $1 \mathrm{~h}$ and their nuclei analyzed by flow cytometry (BD LSRII) using 488-nm excitation and emission collected with a 575/26 band-pass filter. Doublets were identified by a PI signal width versus area plot and excluded from the analysis [36].

\section{Protein detection by Western blot}

$2 \times 10^{7}$ cells were lysed using $200 \mu \mathrm{L}$ lysis buffer (Pierce, Rockford, IL) and lysates were cleared by centrifugation at $13,000 \mathrm{rpm}$ for $30 \mathrm{~min}$ at $4^{\circ} \mathrm{C}$. Equal amounts of protein lysates $(25 \mu \mathrm{g})$ were resolved by SDS-PAGE gel electrophoresis, transferred to PVDF membranes and probed with antibodies. AhR (H211), c-Cbl (C-15) and p Y1021 PDGFR $\beta$ antibodies were from Santa Cruz Biotechnology (Santa Cruz, CA). IRF-1 and CD38 antibodies were from BD Biosciences (San Jose, CA). Antibodies to detect phosphop44/42 MAPK (ERK1/2) (Thr202/Tyr204) (D13.14.4E), p44/42 MAPK (ERK1/2) (137F5), pS221 MEK1/2, MEK1/2, p-Y416 pan-SFK, Lyn, pY507 Lyn, p-PI3K p85(Y458)/p55
(Y199), Fgr, VAV1, p47phox, pS289/296/301 c-RAF (TD cRAF), RAR $\alpha$, and GAPDH, horseradish peroxidase antimouse and horseradish peroxidase anti-rabbit were from Cell Signaling (Danvers, MA, USA). pS621 c-RAF antibody was from Invitrogen (Carlsbad, CA). Cyp1A2 antibody was from Abcam (Cambridge, MA). ECL (GE Healthcare, Pittsburgh, PA) was used for detection.

\section{Statistical analysis}

Statistical analyses were performed using GraphPad (GraphPad software, San Diego, CA). Means of treatment groups of interest were compared using the Paired-Samples $T$ Test. The data represents the means of three repeats \pm S.E.M. A p-value of $<0.05$ was considered significant.

\section{Additional file}

Additional file 1: Figure S1. CD11b Mean Fluorescence Index (MFI) Shift. The flow cytometry raw data and mean fluorescence index for CD11b of a representative experiment (48 h and $72 \mathrm{~h}$ ) are presented. CD11b expression was assessed by flow cytometry with APC-conjugated antibody. HL-60 cells were untreated (C, control) or treated with FICZ, RA, or RA plus FICZ. Cells treated with FICZ alone showed no CD11b expression - like untreated controls.

Competing interests

The authors declare no conflict of interests.

\section{Authors' contributions}

RPB and AY participated in the design of the study. AY coordinated the studies. RPB carried out the experiments. RPB and AY wrote the final manuscript. All authors read and approved the final manuscript.

\section{Acknowledgements}

This work was supported in part by grants R01 CA033505, R01 CA152870 from NIH (A.Y.) and NIH/PS-OC (Shuler), NYSTEM NY Dept. Health (A.Y.), and Cornell Vertebrate Genomics (VERGE) (R.P.B.). The authors would like to thank Holly A Jensen for critical reading of the manuscript.

Received: 7 December 2012 Accepted: 7 May 2013

Published: 9 May 2013

\section{References}

1. Bunaciu RP, Yen A: Activation of the aryl hydrocarbon receptor AhR promotes retinoic acid-induced differentiation of myeloblastic leukemia cells by restricting expression of the stem cell transcription factor Oct4. Cancer Res 2011, 71:2371-2380.

2. Harper PA, Giannone JV, Okey AB, Denison MS: In vitro transformation of the human $A h$ receptor and its binding to a dioxin response element. Mol Pharmacol 1992, 42:603-612.

3. Ohtake F, Baba A, Takada I, Okada M, Iwasaki K, Miki H, Takahashi S, Kouzmenko A, Nohara K, Chiba T, et al: Dioxin receptor is a ligand-dependent E3 ubiquitin ligase. Nature 2007, 446:562-566.

4. Ma Q: Induction of CYP1A1. The AhR/DRE paradigm: transcription, receptor regulation, and expanding biological roles. Curr Drug Metab 2001, 2:149-164.

5. Soprano DR, Soprano KJ: Pharmacological doses of some synthetic retinoids can modulate both the aryl hydrocarbon receptor and retinoid receptor pathways. J Nutr 2003, 133:277S-281S.

6. Shenefelt RE: Gross congenital malformations. Animal model: treatment of various species with a large dose of vitamin a at known stages in pregnancy. Am J Pathol 1972, 66:589-592.

7. Mimura J, Yamashita K, Nakamura K, Morita M, Takagi TN, Nakao K, Ema M, Sogawa K, Yasuda M, Katsuki M, Fujii-Kuriyama Y: Loss of teratogenic 
response to 2,3,7,8-tetrachlorodibenzo-p-dioxin (TCDD) in mice lacking the Ah (dioxin) receptor. Genes Cells 1997, 2:645-654.

8. Hayashida Y, Kawamura T, Hori-e R, Yamashita I: Retionic acid and its receptors are required for expression of aryl hydrocarbon receptor mRNA and embryonic development of blood vessel and bone in the medaka fish, Oryzias latipes. Zoolog Sci 2004, 21:541-551.

9. Hall JM, Barhoover MA, Kazmin D, McDonnell DP, Greenlee WF, Thomas RS: Activation of the aryl-hydrocarbon receptor inhibits invasive and metastatic features of human breast cancer cells and promotes breast cancer cell differentiation. Mol Endocrinol 2010, 24:359-369.

10. Fan Y, Boivin GP, Knudsen ES, Nebert DW, Xia Y, Puga A: The aryl hydrocarbon receptor functions as a tumor suppressor of liver carcinogenesis. Cancer Res 2010, 70:212-220.

11. Ikuta T, Kobayashi Y, Kitazawa M, Shiizaki K, Itano N, Noda T, Pettersson S, Poellinger L, Fujii-Kuriyama Y, Taniguchi S, Kawajiri K: ASC-associated inflammation promotes cecal tumorigenesis in aryl hydrocarbon receptor-deficient mice. Carcinogenesis 2013. doi:10.1093/carcin/bgt083.

12. Boitano AE, Wang J, Romeo R, Bouchez LC, Parker AE, Sutton SE, Walker JR, Flaveny CA, Perdew GH, Denison MS, et al: Aryl hydrocarbon receptor antagonists promote the expansion of human hematopoietic stem cells. Science 2010, 329:1345-1348.

13. Bock KW: The human Ah receptor: hints from dioxin toxicities to deregulated target genes and physiologic functions. Biol Chem 2013, 394:729-739.

14. Takahashi K, Yamanaka S: Induction of pluripotent stem cells from mouse embryonic and adult fibroblast cultures by defined factors. Cell 2006, 126:663-676.

15. Nichols J, Zevnik B, Anastassiadis K, Niwa H, Klewe-Nebenius D, Chambers I, Scholer $\mathrm{H}$, Smith A: Formation of pluripotent stem cells in the mammalian embryo depends on the POU transcription factor Oct4 Cell 1998, 95:379-391.

16. Yu J, Vodyanik MA, Smuga-Otto K, Antosiewicz-Bourget J, Frane JL, Tian S, Nie J, Jonsdottir GA, Ruotti V, Stewart R, et al: Induced pluripotent stem cell lines derived from human somatic cells. Science 2007, 318:1917-1920.

17. Yen A, Roberson MS, Varvayanis S, Lee AT: Retinoic acid induced mitogen-activated protein (MAP)/extracellular signal-regulated kinase (ERK) kinase-dependent MAP kinase activation needed to elicit HL-60 cell differentiation and growth arrest. Cancer Res 1998, 58:3163-3172.

18. Congleton J, MacDonald R, Yen A: Src inhibitors, PP2 and dasatinib, increase retinoic acid-induced association of Lyn and c-Raf (S259) and enhance MAPK-dependent differentiation of myeloid leukemia cells. Leukemia 2012, 26:1180-1188.

19. Shen $M$, Yen A: c-Cbl interacts with $C D 38$ and promotes retinoic acid-induced differentiation and G0 arrest of human myeloblastic leukemia cells. Cancer Res 2008, 68:8761-8769.

20. Yen A, Varvayanis S, Smith JL, Lamkin TJ: Retinoic acid induces expression of SLP-76: expression with c-FMS enhances ERK activation and retinoic acid-induced differentiation/G0 arrest of HL-60 cells. Eur J Cell Biol 2006, 85:117-132.

21. Shen M, Bunaciu RP, Congleton J, Jensen HA, Sayam LG, Varner JD, Yen A: Interferon regulatory factor- 1 binds $\mathrm{c}-\mathrm{Cbl}$, enhances mitogen activated protein kinase signaling and promotes retinoic acid-induced differentiation of HL-60 human myelo-monoblastic leukemia cells. Leuk Lymphoma 2011, 52:2372-2379.

22. Marchisio M, Bertagnolo V, Colamussi ML, Capitani S, Neri LM: Phosphatidylinositol 3-kinase in HL-60 nuclei is bound to the nuclear matrix and increases during granulocytic differentiation. Biochem Biophys Res Commun 1998, 253:346-351.

23. Bertagnolo V, Grassilli S, Bavelloni A, Brugnoli F, Piazzi M, Candiano G, Petretto A, Benedusi M, Capitani S: Vav1 modulates protein expression during ATRA-induced maturation of APL-derived promyelocytes: a proteomic-based analysis. J Proteome Res 2008, 7:3729-3736.

24. Pinel-Marie ML, Sparfel L, Desmots S, Fardel O: Aryl hydrocarbon receptordependent induction of the NADPH oxidase subunit NCF1/p47 phox expression leading to priming of human macrophage oxidative burst. Free Radic Biol Med 2009, 47:825-834.

25. Korn T: How T cells take developmental decisions by using the aryl hydrocarbon receptor to sense the environment. Proc Natl Acad Sci USA 2010, 107:20597-20598.
26. Yen A, Roberson MS, Varvayanis S: Retinoic acid selectively activates the ERK2 but not JNK/SAPK or p38 MAP kinases when inducing myeloid differentiation. In Vitro Cell Dev Biol Anim 1999, 35:527-532

27. Wang J, Yen A: A MAPK-positive feedback mechanism for BLR1 signaling propels retinoic acid-triggered differentiation and cell cycle arrest. J Biol Chem 2008, 283:4375-4386.

28. Dougherty MK, Muller J, Ritt DA, Zhou M, Zhou XZ, Copeland TD, Conrads TP, Veenstra TD, Lu KP, Morrison DK: Regulation of Raf-1 by direct feedback phosphorylation. Mol Cell 2005, 17:215-224.

29. Hekman M, Fischer A, Wennogle LP, Wang YK, Campbell SL, Rapp UR: Novel C-Raf phosphorylation sites: serine 296 and 301 participate in Raf regulation. FEBS Lett 2005, 579:464-468.

30. Ingley E: Functions of the Lyn tyrosine kinase in health and disease. Cell Commun Signal 2012, 10:21.

31. Reiterer G, Bunaciu RP, Smith JL, Yen A: Inhibiting the platelet derived growth factor receptor increases signs of retinoic acid syndrome in myeloid differentiated HL-60 cells. FEBS Lett 2008, 582:2508-2514.

32. Shen $M$, Yen $A$ : c-Cbl tyrosine kinase-binding domain mutant G306E abolishes the interaction of $\mathrm{c}-\mathrm{Cb}$ with $\mathrm{CD} 38$ and fails to promote retinoic acid-induced cell differentiation and G0 arrest. J Biol Chem 2009, 284:25664-25677.

33. Katagiri K, Yokoyama KK, Yamamoto T, Omura S, Irie S, Katagiri T: Lyn and Fgr protein-tyrosine kinases prevent apoptosis during retinoic acid-induced granulocytic differentiation of HL-60 cells. J Biol Chem 1996, 271:11557-11562.

34. Donella-Deana A, Cesaro L, Ruzzene M, Brunati AM, Marin O, Pinna LA: Spontaneous autophosphorylation of Lyn tyrosine kinase at both its activation segment and C-terminal tail confers altered substrate specificity. Biochemistry 1998, 37:1438-1446.

35. Reiterer $\mathrm{G}$, Yen A: Platelet-derived growth factor receptor regulates myeloid and monocytic differentiation of HL-60 cells. Cancer Res 2007, 67:7765-7772.

36. Rinsky RA, Hornung RW, Silver SR, Tseng CY: Benzene exposure and hematopoietic mortality: A long-term epidemiologic risk assessment. Am J Ind Med 2002, 42:474-480.

37. Widerak M, Ghoneim C, Dumontier MF, Quesne M, Corvol MT, Savouret JF: The aryl hydrocarbon receptor activates the retinoic acid receptoralpha through SMRT antagonism. Biochimie 2006, 88:387-397.

38. Kumar MB, Perdew GH: Nuclear receptor coactivator SRC-1 interacts with the Q-rich subdomain of the AhR and modulates its transactivation potential. Gene Expr 1999, 8:273-286.

39. Kamei Y, Xu L, Heinzel T, Torchia J, Kurokawa R, Gloss B, Lin SC, Heyman RA, Rose DW, Glass CK, Rosenfeld MG: A CBP integrator complex mediates transcriptional activation and AP-1 inhibition by nuclear receptors. Cell 1996, 85:403-414.

40. Yang-Yen HF, Zhang XK, Graupner G, Tzukerman M, Sakamoto B, Karin M, Pfahl M: Antagonism between retinoic acid receptors and AP-1: implications for tumor promotion and inflammation. New Biol 1991, 3:1206-1219.

41. Suh J, Jeon YJ, Kim HM, Kang JS, Kaminski NE, Yang KH: Aryl hydrocarbon receptor-dependent inhibition of AP-1 activity by 2,3,7,8tetrachlorodibenzo-p-dioxin in activated B cells. Toxicol Appl Pharmacol 2002, 181:116-123.

42. Klinge CM, Kaur K, Swanson $\mathrm{HI}$ : The aryl hydrocarbon receptor interacts with estrogen receptor alpha and orphan receptors COUP-TFI and ERRalpha1. Arch Biochem Biophys 2000, 373:163-174.

43. Tran P, Zhang XK, Salbert G, Hermann T, Lehmann JM, Pfahl M: COUP orphan receptors are negative regulators of retinoic acid response pathways. Mol Cell Biol 1992, 12:4666-4676.

44. Sassano A, Altman JK, Gordon LI, Platanias LC: Statin-dependent activation of protein kinase Cdelta in acute promyelocytic leukemia cells and induction of leukemic cell differentiation. Leuk Lymphoma 2012, 53:1779-1784

45. Kambhampati S, Li Y, Verma A, Sassano A, Majchrzak B, Deb DK, Parmar S, Giafis N, Kalvakolanu DV, Rahman A, et al: Activation of protein kinase C delta by all-trans-retinoic acid. J Biol Chem 2003, 278:32544-32551.

46. Yen A, Varvayanis S: Retinoic acid increases amount of phosphorylated RAF; ectopic expression of cFMS reveals that retinoic acid-induced differentiation is more strongly dependent on ERK2 signaling than induced GO arrest is. In Vitro Cell Dev Biol Anim 2000, 36:249-255. 
47. Hoyos B, Jiang S, Hammerling U: Location and functional significance of retinol-binding sites on the serine/threonine kinase, c-Raf. J Biol Chem 2005, 280:6872-6878.

48. Hoyos B, Imam A, Korichneva I, Levi E, Chua R, Hammerling U: Activation of c-Raf kinase by ultraviolet light. Regulation by retinoids. J Biol Chem 2002, 277:23949-23957.

49. Mukai R, Shirai Y, Saito N, Fukuda I, Nishiumi S, Yoshida K, Ashida H: Suppression mechanisms of flavonoids on aryl hydrocarbon receptor-mediated signal transduction. Arch Biochem Biophys 2010, 501:134-141.

50. Enan E, Matsumura F: Identification of c-Src as the integral component of the cytosolic Ah receptor complex, transducing the signal of 2,3,7, 8-tetrachlorodibenzo-p-dioxin (TCDD) through the protein phosphorylation pathway. Biochem Pharmacol 1996, 52:1599-1612.

51. Kauss MA, Reiterer G, Bunaciu RP, Yen A: Human myeloblastic leukemia cells $(\mathrm{HL}-60)$ express a membrane receptor for estrogen that signals and modulates retinoic acid-induced cell differentiation. Exp Cell Res 2008, 314:2999-3006.

52. Brooks SC 3rd, Kazmer S, Levin AA, Yen A: Myeloid differentiation and retinoblastoma phosphorylation changes in $\mathrm{HL}-60$ cells induced by retinoic acid receptor- and retinoid $\mathrm{X}$ receptor-selective retinoic acid analogs. Blood 1996, 87:227-237.

doi:10.1186/1476-4598-12-39

Cite this article as: Bunaciu and Yen: 6-Formylindolo (3,2-b)carbazole

(FICZ) enhances retinoic acid (RA)-induced differentiation of $\mathrm{HL}-60$ myeloblastic leukemia cells. Molecular Cancer 2013 12:39.

\section{Submit your next manuscript to BioMed Central and take full advantage of:}

- Convenient online submission

- Thorough peer review

- No space constraints or color figure charges

- Immediate publication on acceptance

- Inclusion in PubMed, CAS, Scopus and Google Scholar

- Research which is freely available for redistribution 